\title{
OTHOTOMICS
}

Revista de economía, empresa y sociedad

Dossier «Prevención de riesgos laborales: tendencias en tiempo de crisis»

ESTRATEGIAS PARA LA PREVENCIÓN

\section{Preservar la salud teletrabajando}

\section{Ines Dalmau Pons}

Consultora de los Estudios de Economía y Empresa de la Universitat Oberta de Catalunya (UOC)

\section{Ramon Ferrer Puig}

Profesor de la Universidad de Barcelona (UB). Departamento de Metodología de las Ciencias del Comportamiento

RESUMEN El teletrabajo supone una manera diferente de llevar a cabo el trabajo. Esto puede generar determinados problemas de salud (tecnoestrés, tecnofatiga, tecnoaddicción, etc.) y quizá evitar otros (lesiones musculoesqueléticas por manipulación de cargas, etc.). Se modifican los factores de riesgo psicosocial que pueden afectar y el papel que tienen. Aspectos como el uso casi constante de la tecnología, la modificación de las relaciones personales, etc., pueden tener una repercusión positiva o negativa. Por lo tanto, en la definición del teletrabajo han de tenerse en cuenta estrategias para prevenir estos posibles riesgos, velar por una óptima gestión del tiempo, disponer de suficientes habilidades comunicativas y de gestión de emociones y un adecuado puesto de trabajo.

PALABRAS CLAVE riesgos psicosociales; prevención; teletrabajo; tecnoestrés; conectividad; disponibilidad

\section{Keeping health while teleworking}

ABSTRACT Telework involves a different approach to working. This can lead to certain health problems (technostress, technofatigue, technoaddiction, etc.) and may prevent others (musculoskeletal injuries due to cargo handling, etc.). The psychosocial risk factors that may affect their role are modified. Aspects like the almost constant use of technology or the changing personal relationships can have a positive or a negative impact. Therefore, the definition of telework must take into account strategies to prevent these potential risks, ensure optimal time management, have adequate emotional management and communication skills and a proper job.

KEYWORDS psychosocial risks; prevention; telework; technostress; connectivity; availability 


\section{Introducción}

Todos queremos gozar de salud, y, además, en el ámbito laboral existe la obligación legal de preservar la salud de los trabajadores. Esto comporta eliminar los riesgos observables y evaluar los que no se pueden eliminar. Actualmente, de una manera u otra, cuando se hace una evaluación de riesgos de una empresa ya se están empezando a identificar los factores de riesgo de tipo psicosocial. Este proceso se está haciendo de manera acelerada, como sucede casi siempre, por el conocimiento de muertos asociados a estos, suicidios en la empresa, asesinatos de usuarios, etc. Los factores de riesgo se están definiendo y conociendo cada vez mejor en los diferentes tipos de trabajo. A continuación se intenta describir cómo cambian estos factores con el teletrabajo, pues, al parecer, este reduce unos elementos de presión, modifica otros e introduce nuevos que quizá hasta su implementación no estaban presentes.

\section{Riesgos psicosociales y teletrabajo}

Aunque se pueden encontrar muchas definiciones de teletrabajo, el elemento en común podría ser «el uso de ordenadores y comunicaciones para cambiar la geografía habitual del trabajo». A partir de esta definición se identifican dos elementos que modifican los posibles factores de riesgo psicosocial de un determinado puesto de trabajo: el uso importante de las tecnologías de la información, un elemento altamente relevante cuando hablamos también de carga mental (Ferrer y Dalmau, 2014), y el hecho de trabajar fuera de los locales o instalaciones de la empresa, lo que cambia del todo el «entorno» de trabajo habitual y también indirectamente puede reducir el tiempo de desplazamiento para llegar al trabajo, con la implicación que esto también tiene en los accidentes in itinere.

A pesar de los puntos en común que hay en las definiciones, un elemento importante que de momento no está del todo consensuado es qué porcentaje de tiempo se considera que cambia la geografía habitual de trabajo. Se habla de teletrabajo a partir del momento en que parte del tiempo se trabaja fuera de las instalaciones de la empresa en tareas que, en principio, se podrían llevar a cabo en estas, pero sin duda no se puede considerar del mismo modo las personas que trabajan fuera un 30\% de su teórica dedicación laboral, que las que están fuera el 90\% del tiempo. «El tiempo de exposición» debe ser tenido en cuenta, del mismo modo que lo es cuando se habla de trabajos con pantallas de visualización de datos (PVD). No se tienen que dejar de lado las características ergonómicas y psicosociales de las tareas cuando se trabaja desde el hotel, el aeropuerto, el bar/restaurante, el autobús, etc.

Si nos fijamos en el área de ergonomía, donde los riesgos son en principio más fáciles de observar que los psicosociales, ya vemos que una cosa es lo que supuestamente va bien y otra lo que realmente en la práctica acabe mejorando, cuando menos todo lo que supuestamente tendría que mejorar.

Cuando se hace una intervención ergonómica, se identifican unos factores de riesgo, se evalúan y se proponen unas medidas que pretenden mejorar. Por ejemplo, en un puesto de trabajo con PVD donde había una silla que solo era regulable en altura, se propone cambiarla por una silla más ergonómica, que tenga cinco regulaciones: altura y profundidad del asiento, altura e inclinación del respaldo y altura de los reposabrazos. Se hace una dotación de cincuenta de estas sillas y al cabo de un mes empiezan de nuevo las quejas: que la silla es mala, que no funciona, etc. Es conocido que el hecho de tener opciones de regulación del entorno físico es mejor, ya que permite precisamente que las personas más altas o bajas tengan una mejor base de sustentación. Pero a pesar de que el dibujito que venía con la silla ya lo indica, de las cincuenta solo en diez se había movido la palanca de profundidad del asiento para ajustarla. Y de estas solo en tres se había movido también la altura del respaldo para ajustarla de acuerdo a las dimensiones del trabajador. Quizá el tener opciones que supuestamente mejoran la ergonomía puede requerir estrategias para valorar cómo pueden afectar a diferentes elementos que están relacionados, y que al modificarlos se puede obtener una afectación diferente a la inicialmente prevista.

A partir de la obligatoriedad legal de prevenir todos los posibles riesgos psicosociales existe la necesidad de intentar identificar cuáles son los posibles «nuevos» problemas de salud que nos podemos encontrar en 
el entorno laboral. Además de los ya conocidos en general, en el ámbito del teletrabajo se empieza a utilizar términos como tecnoestrés, tecnoansiedad, tecnofatiga, nomofobia, tecnoadicción, etc.

De las definiciones de los diferentes términos que están apareciendo, a la vez que se extiende el uso del teletrabajo, destacamos:

- Tecnoestrés: Es un tipo de estrés provocado por la exposición continuada al uso de las nuevas tecnologías de la información y la comunicación (TIC), tales como internet, telefonía móvil, televisión digital, etc.

- Tecnoansiedad: Es el tipo de tecnoestrés más conocido, donde la persona experimenta altos niveles de activación fisiológica no placiente, y siente tensión y malestar por el uso presente o futuro de algún tipo de TIC. La propia ansiedad lleva a tener actitudes escépticas respecto al uso de tecnologías, a la vez que pensamientos negativos sobre la propia capacidad y competencia con las TIC. Un tipo específico de tecnoansiedad es la tecnofobia, que se focaliza en la dimensión afectiva de miedo y ansiedad hacia las TIC (Salanova y otros, 2006).

- Tecnofatiga: Se caracteriza por sentimientos de cansancio y agotamiento mental y cognitivo debidos al uso de tecnologías, complementados también con actitudes escépticas y creencias de ineficacia con el uso de $\mathrm{TIC}$. Un tipo específico de tecnofatiga es el denominado síndrome de la fatiga informativa, derivado de los actuales requisitos de la sociedad de la información y que se concreta en la sobrecarga informativa cuando se utiliza internet. O el multitasking, cuando se distrae temporalmente la atención de la tarea principal hacia otras actividades para volver posteriormente a la tarea inicial. En este caso, el cerebro se ocupa a la vez de todos estos requerimientos extras, en espera de ser resueltos.

- Tecnoadicción: Es la adicción a las nuevas tecnologías. Es una adicción como cualquier otra, definida como la necesidad hacia algo que proporciona bienestar y placer; así, como las nuevas tecnologías generan cierta satisfacción, se quiere pasar la mayor parte del tiempo interactuando con ellas (Rosen, 2013). Está relacionada con la neofilia, que tiene como principal característica la necesidad de tener siempre lo último en tecnología, la compra compulsiva de aparatos tecnológicos y software actualizado.

- Nomofobia: Miedo irracional a estar sin teléfono móvil. El término proviene del anglicismo nomophobia (no-mobile-phone-phobia). La dependencia al dispositivo electrónico genera una infundada sensación de incomunicación en el usuario cuando este no puede disponer de él. Similar al concepto de fomo, que proviene de la expresión inglesa fear of missing out, que quiere decir 'miedo a estar desconectado'.

En los medios de comunicación se empieza a utilizar el término e-nomads, personas en las que la tecnología ha invadido su vida profesional y personal con un horario de trabajo que no tiene hora de fin establecida; la carga de trabajo y la complejidad de tareas se multiplica en proporción al número de tecnologías utilizadas; se pierde el sentido de selección de información por la ilimitada cantidad de datos disponible; las relaciones personales se sustituyen por relaciones virtuales, y el balance de vida laboral y profesional es imposible de establecer porque los dos aspectos están mezclados. Así todos los días. Además, se pueden generar desórdenes musculoesqueléticos derivados de las inadecuadas posturas que se adoptan al utilizar los dispositivos en cualquier momento y lugar.

Se tendrá que ir viendo, con el avance en el estudio de estas temáticas, cuáles de estos términos acaban siendo realmente considerados como posibles riesgos psicosociales, más prevalentes, aunque no exclusivos, del teletrabajo.

\section{Factores de riesgo. Aspectos positivos y negativos del teletrabajo}

Actualmente no se dispone de una lista exhaustiva ni consensuada de los factores de riesgo psicosocial. Aunque se tuviera, se sabe que, del mismo modo que en los factores ergonómicos, lo que se busca es la adaptación a la persona, ni demasiado alto ni demasiado bajo, ni demasiado ni demasiado poco, lo que se intenta conseguir es buscar referentes que nos puedan orientar hacia cuál puede ser el punto adecuado en cada caso. Además, 
cuando se modifica un factor se puede estar incidiendo en cómo afecta a otro u otros, que también se tendrán que ajustar a esta nueva situación. En general, los riesgos psicosociales son multifactoriales y encontrar el punto adecuado implica tener en cuenta la mejor combinación de varios factores a la vez, puesto que el que puede funcionar en un caso no tiene por qué funcionar en otro (EASHW, 2008; EASHW, 2012; Departamento de Gobernación y Administraciones Públicas, 2008).

\subsection{Aspectos importantes para el trabajador}

En este apartado se describen algunos de los elementos que cambian y que tienen un impacto en la persona que teletrebaja (Rubini, 2012; Pol y otros, 2013; Morell, 2007; Baraza y otros, 2014).

- Horario laboral flexible, adaptable a las necesidades personales; ahora bien, ¿se prolonga o no la disponibilidad personal?

- Mejora la conciliación entre la vida laboral y la profesional, pero ¿hay que hacer un tipo de pacto familiar/ social?

- Más autonomía y control sobre el propio trabajo y entorno social. ¿Cómo se gestionan las llamadas no respondidas en el momento?

- Se reducen los desplazamientos, se ahorra tiempos. También se reducen los contactos sociales que se hacen en estos desplazamientos y este tiempo a veces también se aprovecha para trabajar.

- Se puede trabajar en otros lugares: hoteles, aeropuertos, bares, segunda residencia, etc. Se puede trabajar en un entorno no muy adecuado, sin tener todos los recursos necesarios, pero intentando cubrirlos.

- Se puede seleccionar el lugar de residencia independientemente de la ubicación del trabajo. Seguramente hasta cierto punto.

- Si se sufre alguna discapacidad, se puede trabajar en un entorno adaptado personal. Supuestamente más adaptado.

- La globalización, las nuevas tecnologías y las políticas europeas fomentan cada vez más esta modalidad de trabajo. ¿También el deseo social? No parece claro si socialmente es deseable no trabajar en un despacho, con compañeros cercanos y fácilmente accesibles para consultas, quizá ocuparse de más cosas de casa, etc.

- Es imprescindible ser capaz de organizarse el tiempo, parece que se debe tener una gran autodisciplina y motivación hacia el trabajo. ¿Se mantienen los hábitos saludables: dormir, comer, etc.? ¿La persona es capaz de soportar el aislamiento? ¿Y de separar la vida laboral de la familiar? Gran impacto en las relaciones sociales; parece claro que no todo el mundo sirve.

- Conviene tener un buen conocimiento de informática. Una actitud receptiva hacia las nuevas tecnologías. ¿Sabemos lo suficiente como para desde casa solucionar los problemas? ¿Nos acostumbramos a tantos cambios, a veces no del todo explicados? ¿El software que utilizamos es el que se adapta mejor a nuestras necesidades?

- Es mejor tener un espacio específico, adecuado e independiente para teletrabajar. Probablemente comporte una inversión económica: espacio, módem, conexión a internet, luz, silla adecuada, software, etc.

\subsection{Aspectos importantes para la empresa}

Por otro lado, en este apartado se describen algunos de los elementos que inicialmente tienen un impacto para la empresa con personas que teletrabajan (Morell, 2006; Baraza, 2014).

- Reducción de costes especialmente en infraestructuras. Dependerá del grado de teletrabajo. Si el trabajo es en cooperación, ¿cuánto será el tiempo perdido para que todos participen?

- Parece que un incremento de la productividad no contabiliza el tiempo de «descanso", menos interrupciones directas. ¿Y el ahorro en las soluciones en la conversación del pasillo, tomando un café?

- Facilita la contratación de personal, se reducen las limitaciones por razón del lugar de residencia. Hasta cierto punto. 
- Hay una mayor dificultad en el control de la evolución del trabajo. Se tiene menos conocimiento del proceso, importante si solo se consideran los resultados. Se puede ver afectada la motivación. ¿Se mantienen las oportunidades de promoción individual?

- Se puede reducir el sentimiento de pertenecer a una determinada organización. Puede incrementar la distancia entre personal y empresa. ¿Se mantienen del mismo modo la cultura, los valores empresariales?

- Todavía hay un vacío legal y fiscal, aspectos de responsabilidades laborales, responsabilidades en la confidencialidad de datos, etc.

- Se mejora considerablemente el clima laboral y la satisfacción profesional. En relación con este punto parece que de momento hay resultados cuestionables, incluso contradictorios.

\section{Orientaciones para la intervención preventiva}

Teniendo en cuenta el punto anterior, algunas de las estrategias para prevenir los factores de riesgo psicosocial asociadas al teletrabajo tendrían que estar relacionadas con los siguientes elementos (Salanova, 2006; Departamento de Gobernación y Administraciones Públicas, 2008; Rosen, 2013; Pol y otros, 2013):

\subsection{Gestión del tiempo}

Estrategias que permiten tener un control del tiempo real de trabajo: cuantificar el tiempo que se está disponible para trabajar, tener conocimiento y diferenciar las horas que se está trabajando y las que no se está trabajando.

Diferenciar en la jornada laboral el tiempo de reacción del tiempo efectivo. En muchos trabajos se tienen unas tareas que supuestamente se tienen que hacer y otras que vienen sobrevenidas. Esta medida consiste en prever, a partir de la experiencia el tiempo que se necesita, y reservar el tiempo necesario para poder atender adecuadamente las tareas sobrevenidas. Por ejemplo, tiempo entre reunión y reunión.

Priorizar tareas adecuadamente, diferenciando entre urgente e importante y definiendo el grado real de urgencia. Que haya llegado ahora un mensaje de correo no tiene por qué querer decir que se tenga que contestar ahora. Es necesario facilitar la concentración en una tarea concreta. Hay que dar orientaciones claras.

\subsection{Habilidades comunicativas}

En el caso del teletrabajo, se modifica la manera como se tienen las relaciones interpersonales. La distancia física y emocional con las personas del entorno laboral se suele incrementar, por lo que se ha de intentar preservar.

Modelos de comunicación efectiva. Con las nuevas tecnologías se ha incrementado el uso del lenguaje escrito. Las características de este tipo de lenguaje son diferentes a las verbales, entre otras cosas requiere una mayor precisión en las palabras y la consideración de la llamada netiqueta. Se pueden facilitar modelos y criterios.

Mantener la escucha activa. Todo lo que se suele recomendar en cuanto a escucha activa en los cursos relacionados con la comunicación se hace más necesario en el caso del trabajo a distancia. No se pueden dar por entendidas las cosas, se tendrán menos indicadores de cómo están siendo entendidas. Prever un adecuado seguimiento del proceso.

Optimizar la gestión de reuniones. Habitualmente el tiempo que se comparte en el entorno de trabajo cuando se teletrebaja se aprovecha para realizar reuniones, actividades en las que se requiere la presencia simultánea de varias personas. Como estas son más escasas, es importante que estén el máximo de preparadas posible. Que tengan objetivos claramente definidos, limitación temporal, tareas que se deriven de ellas, etc.

\subsection{Gestión de emociones}

Relacionado con el punto anterior, en el caso del teletrabajo se modifica la manera como se tienen las relaciones interpersonales, y esto puede tener una repercusión en las estrategias personales de gestión emocional. 
Mantener contactos afectivos. Para cualquier persona es necesario sentir emociones relacionadas con recibir y dar afecto. A pesar de que la mayoría de las personas tienen estas necesidades más o menos cubiertas en el ámbito privado, el trabajo puede ser un lugar donde también se produzca este intercambio, y para determinadas personas puede ser prácticamente el único entorno donde los tienen o el más importante. Hay que buscar estrategias para poder mantener estas vivencias positivas también en el trabajo. Facilita el mantener el sentimiento de pertenencia.

Prever la gestión de posibles conflictos. La realidad de las relaciones interpersonales nos permite prever que en un grupo de personas probablemente aparecerá algún conflicto. Se tendría que anticipar de qué manera se podrá facilitar su resolución.

\subsection{Puesto de trabajo}

A pesar del actual vacío legal de algunos de estos aspectos, especialmente en relación con las características de un entorno privado, se tendría que cumplir la normativa vigente en materia de prevención de riesgos, medio ambiente, confidencialidad de datos, derechos laborales, etc.

En la medida de lo posible se deben seguir las recomendaciones publicadas por organismos acreditados. Entre otros, prevenir los riesgos ergonómicos, disponer de toda la tecnología necesaria, valorar la adecuación del software que se utiliza, definir funciones y responsabilidades, identificar costes, etc.

\section{Conclusiones}

El teletrabajo es una manera de organizar el trabajo que cambia sustancialmente las condiciones de trabajo, modifica los factores de riesgo psicosociales presentes. Se están dando casos de personas que manifiestan problemas de salud relacionados con el uso de las nuevas tecnologías, tecnoestrés, tecnofatiga, tecnoadicción, etc. La posibilidad del teletrabajo va asociado al uso de estas tecnologías junto con el cambio de otras condiciones de trabajo, por lo que puede ser un elemento que incremente los daños en la salud.

El impacto de estos factores, como siempre, es diferente según las personas, lo que la psicología denomina diferencias individuales. El hecho de no tener bien definido cuándo estoy trabajando y cuándo no en sí mismo es un factor que incide en el nivel de estrés. A priori es una ventaja para la persona no tener que desplazarse al centro de trabajo, pero no queda del todo claro si esto incrementa el hecho de estar más tiempo disponible por temas de trabajo y el aislamiento sociolaboral. Entre otros aspectos, el tiempo y el tipo de relaciones laborales también cambia.

Cuando una empresa opta por impulsar el teletrabajo en su organización, ha de tener en cuenta estrategias que permitan controlar y reducir los posibles efectos negativos que puede generar tanto en las personas como en las organizaciones, así como potenciar los efectos positivos. Es necesario hacer un seguimiento, tener evaluado el impacto real que está teniendo, y en su caso proponer y realizar los cambios o ajustes necesarios. 


\section{Bibliografía}

BARAZA, X.; RIMBAU-GILABERT, E.; DE LA DEHESA, J.; MORRAL, B.; RUIZ, R. (2014). «Influencia del modelo de liderazgo en la cultura organizacional y la prevención de riesgos psicosociales». Proceedings of the 12th International Conference on Occupational Risk Prevention [artículo en línea]. http://www.orpconference.org

EUROPEAN AGENCY FOR SAFETY AND HEALTH AT WORK (2008). «Risk assessment for teleworkers». E-FACTS. 33 [artículo en línea]. https://osha.europa.eu/en/publications/e-facts/efact33.

EUROPEAN AGENCY FOR SAFETY AND HEALTH AT WORK (2008). «Management of psychosocial risk at work: an analysis of the findings of the european survey of enterprises on new and emerging risks ESENER)". Luxemburgo: EU-OSHA [artículo en línea]. https://osha.europa.eu/en/publications/reports/managementpsychosocial-risks-esener/view

FERRER, R.; DALMAU, I. (2014). «Ergonomía cognitiva y carga mental». En: GIL-MONTE, P. (coord.). Manual de psicosociología aplicada al trabajo y a la prevención de los riesgos psicosociales. Madrid: Pirámide.

Morell, J. (2007). «Teletreball. Quan el treball deixa de ser en un lloc». Departament de Cibersocietat de la Fundació iBit. Monografia 6. [artículo en línea]. http://obsi.fundaciobit.org/wp-content/uploads/2012/06/Monografia-6Teletreball.pdf.

POL, E.; NET, E.; FERRER, R. (2013). «L'environnement du télétravailleur». En: RIOUX, L.; LE ROY, J.; RUBENS, L.; LE CONTE, J. (eds.). Le confort au travail. Que nous apprend la psychologie environnementale? París: Presses de l'Université Laval.

DEPARTAMENT DE GOVERNACIÓ I ADMINISTRACIONS PÚBLIQUES (2008). Guia per a la introducció al teletreball a l'Administració de la Generalitat de Catalunya [en línea]. http://www.ccoo.cat/fsap/s_gene/ ccoogene/documents/teletreball/Guia_introduccio_teletreball.pdf

ROSEN, L. D. (2013). iDisorder: Understanding Our Obsession with Technology and Overcoming Its Hold on Us. Londres: MacMillan.

RUBBINI, N. (2012). «Los riesgos psicosociales en el teletrabajo». VII Jornadas de Sociología de la Universidad Nacional de La Plata [artículo en línea]. http://jornadassociologia.fahce.unlp.edu.ar/actas/Rubbini.pdf

SALANOVA, M.; LLORENS, S.; CIFRE, E.; NOGAREDA, C. (2006). «Tecnoestrés: concepto, medida e intervención psicosocial. NTP 730. Madrid: INSHT [artículo en línea]. http://www.insht.es/InshtWeb/Contenidos/ Documentacion/FichasTecnicas/NTP/Ficheros/701a750/ntp_730.pdf

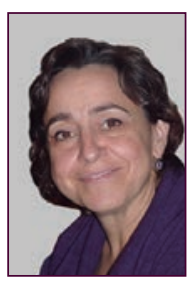

\section{Ines Dalmau Pons}

\section{idalmaup@uoc.edu}

Consultora de los Estudios de Economía y Empresa de la Universitat Oberta de Catalunya (UOC)

Doctora en Organización y Administración de Empresas por la UPC. Máster en Ergonomía por la UPC. Máster en Prevención en las tres especialidades. Licenciada en Psicología por la UB. Consultora de los Estudios de Economía y Empresa de la UOC. Profesora por la UB en el Máster universitario en seguridad y salud en el trabajo. Prevención de riesgos laborales (UB-UPC-UPF). Especialista del Área de Ergonomía y Psicosociología de un Servicio de Prevención Mancomunado que da servicio a unos 5.000 trabajadores. Autora de varias publicaciones de psicosociología, ergonomía, carga mental y prevención de riesgos laborales. 


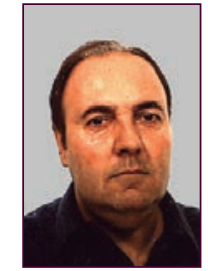

Ramon Ferrer Puig

ramon.ferrer@ub.edu

Profesor de la Universidad de Barcelona (UB). Departamento de Metodología de las Ciencias del Comportamiento

Doctor en Psicología (UB). Profesor titular del Departamento de Metodología de las

Ciencias del Comportamiento (UB). Máster en Seguridad y gestión del riesgo industrial (UPC). Técnico superior de PRL en las tres especialidades. Autor de varias publicaciones sobre prevención de riesgos laborales.

Los textos publicados en esta revista están -si no se indica lo contrario- bajo una licencia Reconocimiento-Sin obras derivadas 3.0 España de Creative Commons. Puede copiarlos, distribuirlos y comunicarlos públicamente siempre que cite su autor y la revista y la institución que los publica (autoría, nombre de la revista, institución editora); no haga con ellos obras derivadas. La licencia completa se puede consultar en http://creativecommons.org/licenses/by-nd/3.0/es/deed.es.

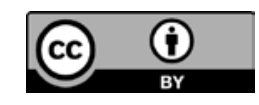

